\title{
When marks become graphics. Rediscovering a forgotten Bertin's fork?
}

\author{
Gilles Rouffineau \\ ESAD・Grenoble•Valence, France, gilles.rouffineau@esad-gv.fr,
}

Keywords: Jacques Bertin, Graphics, Education, Logarithmic matrix, Visualisations.

\begin{abstract}
:
Adaptations of Semiology of Graphics: Diagrams, Networks, Maps (Bertin, 1967), and more broadly Jacques Bertin's graphics research published since the mid-sixties, are manifold. So is the wide range of fields chosen to present various visual transformations and deep interpretations proposed to explain his actual graphical methods. From agriculture to demography, or european electric industry to animal behaviour responding to the light (pill bugs...), anything that can be quantified, compared and classified could fit in some graphic treatment for a better understanding. In this respect, graphics is able to go deeper and faster than any other analysis.
\end{abstract}

I would like to present a forgotten, unusual, rather unfinished, attempt to make use of graphics in a french design graduate school pedagogy during the eighties. Obviously, the impact of Bertin's research is huge in the cartography and social or historical sciences, but it seems seldom in the more casual educational domain, and more particularly in graphic design training course. Is it a paradox?

Coming first in the state of art, Roberto Gimeno's book Apprendre à l'école par la graphique (Gimeno, 1980) (i.e. "Learning in primary school with graphics") published in french language, is a noteworthy exception. It is based on real experiments and described several activities in the classroom for very young childrens. This educational adaptation is probably not unique but really outstanding because of the closeness with the main founder on the field theory and also for it's rigorous and canonical methodology following Bertin's rules, particularly the "orderable matrix". The main goal of this work provides a clear demonstration that, with well guided context, school childrens are able not only to understand Bertin's methodology by doing, but also to gain new insights at their proper level.

Beside Gimeno's quite singular experiments, I had the opportunity to met, in the early eighties an other Bertin's close collaborator then teaching design culture and communication in Valence Art and Design School : Michel Curtenaz. First trained as a photographer, he worked later on advertising and communication business as a writer and advisor. Deeply involved in Bertin's activities, he gave to the students - and all our teaching team - the very first introduction to Bertin's graphics theory and practices. Today, a lost bag with "domino 3", unused matrix material, still overlooked is waiting somewhere in a wall shelf of the school. As far as I know, unfortunately Curtenaz did not write any academic text about his research, and his actual relationship with Bertin's laboratory is not easy to established nowadays. All I could remember quite well is the story of a mandat he had contracted to give informations helping the french Ministry of Interior to priorise subsidies allowed for farmers suffering after severe drought in 1976 summer. This assignment, not so far to an intelligence task, required graphics made from aerial pictures to give the right grant level responding to the real harvest disease. Nothing could really be said nor written about that mission. In this case, graphics had some very political issues.

After Curtenaz's death in 1999, part of his personal documentation and specialised professional library has been kept in the records of the school, as compiling an archival memory responding some friends and family request. In an archaeological perspective, some notes, from classroom trainings including Bertin's teachings and most surprisingly, various issues of school report from this early period are recorded. Graphic design students were starting new courses with new professional diploma two years program : D.N.A.T. (Diplome National d'Arts et Techniques). The very first quarter shows graphical marks not as usual numbers, in a range from 0 to 20 , but as black rectangles drawn from various levels for each topics and criteria. (Fig. 1)

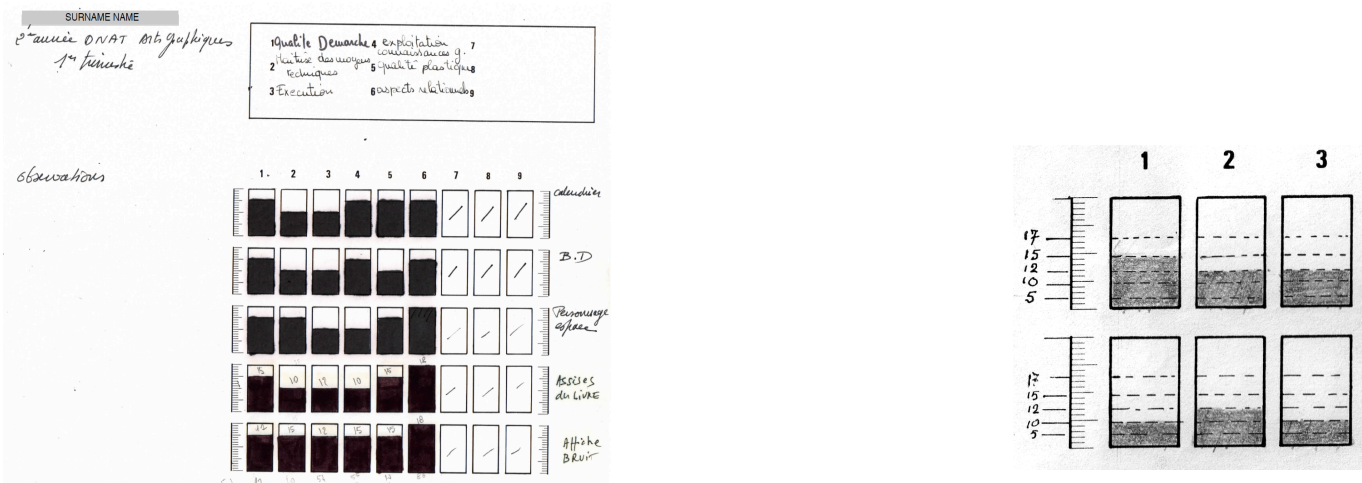

Fig. 1 - School report, first quarter 1982-1983. Fig. 2 - Logarithmic scaling marks from 5 to 17, 2nd quarter. (detail) 
Clearly inspired by Bertin's practices, these graphics are quite unfamiliar and striking for scholarly reports, but also a bit confusing. Let's look closer at this charts. We can read in the first set of form, issued from the first quarter in 1982-1983 academic year, that students have been involved in five different works : Calendar, Comics, People in space, Library event and Poster about "noise" subject. The value was splitted into six criteria : 1/ Quality of process, 2/ Technical skills, 3/ Execution, 4/ Knowledge exploitation, 5/ Visual qualities, and more oddly 6/ Relationship aspects. Beyond any considerations on topics and criteria more or less relevant in this fresh graphic design course, let's consider the way visual forms give informations as a level of success. The only variable size (height) was used.

At this very first stage, the form is clearly still a blue print. Some numbers remains despite graphics should replace marks. Moreover, different marks are supposed to give visual different levels, but in this form, different values have the exact same black drawn surface. Sometimes 10 is the same as 12 , which is also equal to 15 elsewhere with an other rate. Of course, this is not a final report, just a messy sketch. But more important, going through all the other students forms, it seems that graphical process was just starting to established Bertin's first self training in the class room. Various hand writings, probably from each students, tell us that it could probably be more a trial sheet, or an exercice, than a final and legal form to communicate a valid result. What seemed at first glance be an academic form must be seen as teaching sketches. These sheets are only the very first draft step to learn Bertin's scaling practices.

From the same academic year, second quarter set shows a more accurate scaling, with logarithmic guide lines. (Fig 2) We can notice that black marker has been replaced with pencil, allowing to fix possible mistakes more easily. Now, all numbers have disappeared but the scale itself, so the process, is really improved in a more flexible and graphical way. All criteria (outside of the frame in Fig. 2) are no longer hand writted but typewritten. The third and last quarter of the year is rather similar to the second one. At the end of the academic year, a new form summarise the full academic results. (Fig. 3)



Fig. 3 - Final school report, full academic year 1982-1983.



Fig. 4 - A new logarithmic scaling, 1983-1984.

Although incomplete - third trimester was left blank on bottom - this principle could allow more abstract comparisons and progression all the year along. All the assignments have let the place for a unique and global quarter mark, and previous criteria have merged in three main topics : Skills, Theory and Style. Relationship, that means the ability to have exchanges with other students and teachers, the involvement in the "group dynamic", are still alone and very important on the right side. Fine art research, English and Legal informations, such as company law or copyright are now present all the year long. Number marks are coming back in this graphic, probably before they should be erased. The next year, with the same group of students, an other scaling draft is provided, a bit different according to it's progression. The new one used only even numbers instead of the previous odd and even numbers mix. (Fig. 4 \& 2).

What can be said to conclude this brief historical excavation? There is no doubt, knowledge and practices of Bertin's graphics theory are on the background of this educational process with the aim to give more visibility for scaling marks in a visual and direct way. Today we are still missing students testimonials, but I've heard from my friend Michel Curtenaz that he find this experience mostly unsatisfactory, if not a teaching failure. But although the results are not really compelling, it's an interesting attempt to develop a new visual application with quite simple but non-linear chronograms. Can we say that graphic design field should be the worth one for this kind of project?

As suggests Bertin many times : «Graphics and graphism are two crucially different languages according to their goals. Their confusion who drives to misleading conventions is the biggest graphical mistakes source. » (Bertin, 1977, p. 178)

Bertin J. (1967), Semiology of Graphics: Diagrams, Networks, Maps, University of Wisconsin, [1983] trans. Sémiologie graphique, les diagrammes, les réseaux, les cartes, Paris, Gauthiers-Villars.

Bertin J. (1977), La graphique et le traitement graphique de l'information, Paris, Flammarion.

Bonnin, S. (1975), Initiation à la graphique, Paris, EPI éd.

Gimeno R. (1980), Apprendre à l'école par la graphique, Paris, Retz. 